\title{
Maximum-likelihood estimation of emission and attenuation images in 3D PET from multiple energy window measurements
}

\author{
Ludovica Brusaferri, Student Member, IEEE, Alexandre Bousse, Yu-Jung Tsai Student Member, IEEE, David \\ Atkinson, Sébastien Ourselin, Brian F. Hutton, Senior Member, IEEE, Simon Arridge and \\ Kris Thielemans, Senior Member, IEEE
}

\begin{abstract}
This study explores the feasibility of incorporating energy information into a maximum-likelihood reconstruction of activity and attenuation (MLAA) framework. The attenuation and activity distributions were reconstructed from multiple energy window data, and a scatter function was added to the system model of the algorithm. The proposed energy-based method (MLAA-EB) was evaluated with simulated 3D phantom data, using the geometry and characteristics of a Siemens mMR PETMR scanner. Results showed that the proposed algorithm is able to compensate for errors in the activity image caused by the incorrect assignment of attenuation values to the segmented MR. This is effective for small objects only, for large objects further solutions need to be found.
\end{abstract}

\section{INTRODUCTION}

$\mathbf{T}$ He problem of attenuation correction of PET images represents a challenging issue in PET/MR scanners, as it is not possible to directly correlate the MR signal to tissue attenuation of the $511 \mathrm{keV}$ photons.

Therefore, emission-based attenuation estimation strategies seem to be particularly promising for overcoming the quantification errors induced by conventional MR-based attenuation correction methods [1] in PET/MR. However, in the absence of time-of-flight information, the joint reconstruction of the activity and attenuation map is a strongly ill-posed problem with infinitely many solutions. Therefore, alternative approaches need to be found.

Berker et al [2] investigated the possibility of incorporating the scatter information into a joint reconstruction scheme.

Manuscript received December 13, 2018. This work is supported by Siemens Healthineers, the UCL Impact Scheme, NIHR UCLH Biomedical Research Centre, the EPSRC-funded UCL Centre for Doctoral Training in Medical Imaging (EP/L016478/1) and UCL Institute of Healthcare Engineering.

Ludovica Brusaferri is with the Institute of Nuclear Medicine, University College London, London NW12BU, UK (e-mail: 1.brusaferri.16@ucl.ac.uk) Alexandre Bousse was with the Institute of Nuclear Medicine, University College London, London NW1 2BU, UK.

Yu-Jung Tsai is with the Institute of Nuclear Medicine, University College London, London NW1 2BU, UK.

David Atkinson is with the Centre for Medical Imaging, University College London, London W1W 7TS, UK.

Sébastien Ourselin is with the Department of Medical Physics and Biomedical Engineering, University College London, London WC1E 6BT, UK.

Brian Hutton is with the Institute of Nuclear Medicine, University College London, London NW1 2BU, UK.

Simon Arridge is with the Department of Computer Science, University College London, London WC1E 6BT, UK

Kris Thielemans is with the Institute of Nuclear Medicine, University College London, London NW1 2BU, UK (e-mail: k.thielemans@ucl.ac.uk).
Whilst promising, the assumption that photopeak and scattered data can be measured separately represents a limitation for this approach. The study is also restricted to small 2D phantoms representing a mouse-sized object.

In previous work, we demonstrated that the finite energy resolution of PET detectors can be efficiently modelled when using multiple energy window measurements and that it possible to estimate the attenuation distribution of the object from such data if the emission image is known [3]. This paper explores the feasibility of incorporating a 3D probabilistic scatter model into a joint reconstruction scheme, without having to resort to the assumption of the absence of scatter in the photopeak window. We also provide a preliminary evaluation of the performance of the method on simulated data.

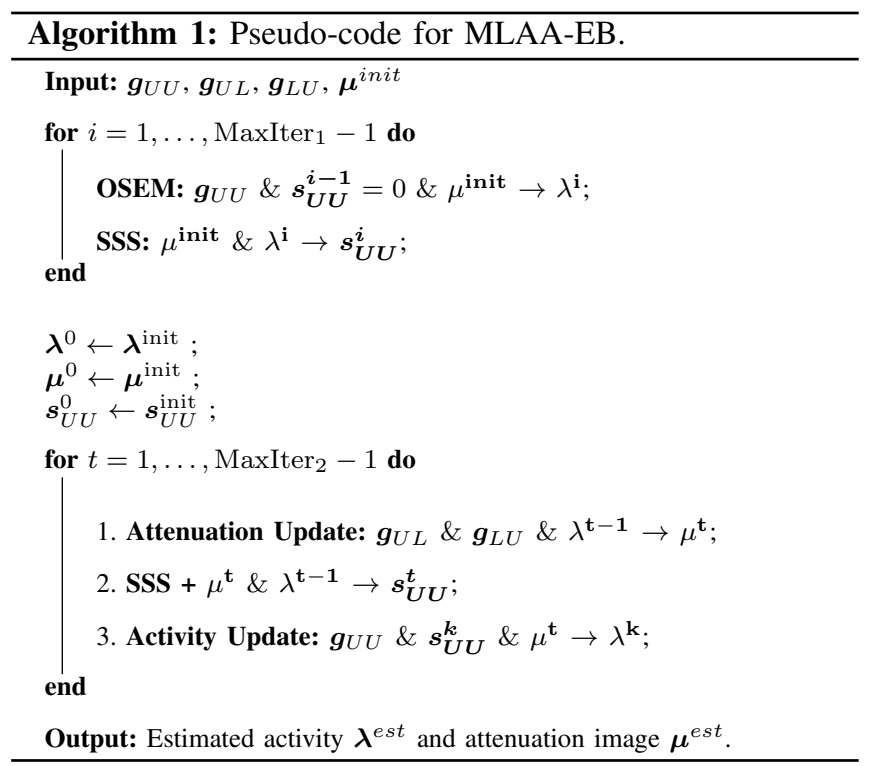

\section{Methods}

\section{A. Multiple Energy Window Data}

In the proposed method, it is assumed that each detector can assign a photon to either the photopeak window (U) or a lower energy window (L) placed below the photopeak. Therefore, four different sinograms are created, one for each combination: $\mathbf{g}_{\mathbf{U U}}, \mathbf{g}_{\mathbf{U} \mathbf{L}}, \mathbf{g}_{\mathbf{L U}}, \mathbf{g}_{\mathbf{L L}}$. We assume that the observed counts $\mathbf{g}$ 
are given by the sum of unscattered and scattered events. In our current method, we disregard the presence of unscattered events in the scatter windows. This leads to:

$$
\begin{gathered}
g_{U U}=g_{U U}^{u n s c}+g_{U U}^{s c} \\
g_{U L} \approx g_{U L}^{s c} \\
g_{L U} \approx g_{L U}^{s c}
\end{gathered}
$$

We currently assume that $\mathbf{g}_{\mathbf{U}}^{\mathrm{sc}}, \mathbf{g}_{\mathbf{U L}}^{\mathrm{sc}}$ and $\mathbf{g}_{\mathbf{L U}}^{\mathrm{sc}}$ contain single scatter events only. We do not use the $\mathbf{g}_{\mathbf{L L}}$ data as it contains very few single scatter events [3].

The expected number of unscattered counts $\overline{\mathrm{g}}_{\mathbf{U} \mathbf{U}}^{\text {uns }}$ in the photopeak window $\mathbf{U} \mathbf{U}$ is defined for each pair of detectors $i j$ as:

$$
\overline{\mathbf{g}}_{\mathbf{U U}, \mathbf{i j}}^{\text {unsc }}=\epsilon_{\mathbf{U}}(\mathbf{5 1 1}) \epsilon_{\mathbf{U}}(\mathbf{5 1 1})\left(\mathbf{G}_{\mathbf{i j}} \lambda\right) \mathbf{e}^{-\mathbf{G}_{\mathbf{i j}} \mu(\mathbf{5 1 1})}
$$

where $\epsilon_{w}$ and $\epsilon_{y}$ denote the detection probability of a photon with a certain energy in a given energy window, $\lambda$ and $\mu$ represent the activity and the attenuation distributions respectively, $\mathbf{G}_{\mathbf{i j}}$ is the projector operator along the line of response (LOR) spanned by two detectors $i, j$.

The expected number of scatter counts $\overline{\mathbf{g}}_{\mathbf{U L}, \mathbf{i j}, \mathbf{S}}^{\mathbf{s c}}$ in the energy window pair $\mathbf{U L}$ (and similarly in $\mathbf{L U}$ ) is computed using an extension of the single scatter simulation (SSS) [4] to the case of multiple energy window measurements:

$$
\begin{gathered}
\overline{\mathbf{g}}_{\mathbf{U L}, \mathbf{i j}, \mathbf{S}}^{\mathbf{s c}} \propto \\
\epsilon_{U}(E) \epsilon_{L}(511) \mu(511, S)\left(\mathbf{K}_{\mathbf{i S}} \lambda\right) \mathbf{e}^{-\mathbf{K}_{\mathbf{i S}} \mu(\mathbf{5 1 1})} \mathbf{e}^{-\mathbf{K}_{\mathbf{j} \mathbf{S}} \mu(\mathbf{E})} \\
+\epsilon_{U}(511) \epsilon_{L}(E) \mu(511, S)\left(\mathbf{K}_{\mathbf{j} \mathbf{S}} \lambda\right) \mathbf{e}^{-\mathbf{K}_{\mathbf{j} \mathbf{S}} \mu(\mathbf{5 1 1})} \mathbf{e}^{-\mathbf{K}_{\mathbf{i} \mathbf{S}} \mu(\mathbf{E})}
\end{gathered}
$$

where $S$ is the scatter point, $\mathbf{K}_{\mathbf{i S}}$ and $\mathbf{K}_{\mathbf{j S}}$ denote the projector operator along the line connecting each detector with the scatter point, respectively $i S$ and $j S, E$ is the scattered photon energy, and $\mu(E)$ is the attenuation coefficient at the energy E.

The model also accounts for the fact that Compton Scattering is not equally probable at all scattering angles, as described by the Klein-Nishina equation [5]. It also considers the detector cross-sections normally presented to the rays $j S$ and $k S$. These factors are omitted from Eq. 5 for simplicity of notation.

\section{B. MLAA-EB Optimisation algorithm}

The proposed MLAA-EB optimisation algorithm (EnergyBased Maximum Likelihood reconstruction of attenuation and activity) relies on alternating maximisation in $\hat{\lambda}$ and $\hat{\mu}$.

The emission image is reconstructed with a Maximum Likelihood (ML) estimator from UU data, whilst the attenuation image is obtained with the MLTR-EB algorithm [3] from the other energy window pairs (UL, LU). Both likelihoods use a Poisson model.

The algorithm alternates between estimating activity from UU data (for a maximum of 3 iterations) and attenuation from UL and UL data (for a maximum of 1 iteration), where each step uses LBFGS-B [6]. The gradient $\nabla_{\mu}\left(\mathbf{g}^{\mathbf{U L} / \mathbf{L U}} \mid \lambda, \mu\right)$
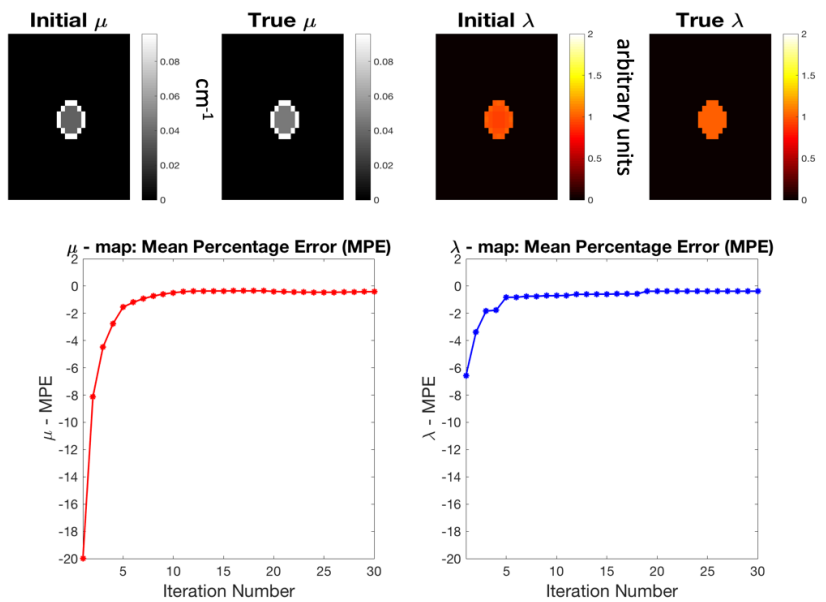

Fig. 1. Small cylinder $(D=8 \mathrm{~cm})$. First row: Initial attenuation image $(20 \%$ error in the inner cylinder), true attenuation image, initial and true activity images. Second row: mean percentage error over iteration number, for the attenuation and activity images.

was derived analytically from the forward scatter model, implemented in STIR [7] and validated with finite differences.

Both the forward model and the gradient account for the dependency of the energy attenuation of the photon as well as the finite energy resolution of the PET detectors.

The scatter component in the photopeak UU is computed from the current estimate of the attenuation image and the emission data, using an extension of the single scatter simulation (see Eq. 5)

Algorithm 1 shows the pseudo-code for the proposed reconstruction algorithm.

\section{Simulation Set-Up}

Simulations have been performed using the geometry and characteristics of the Siemens mMR scanner [8]. The lower energy window range was set to $\mathrm{L}=350-460 \mathrm{keV}$ and the upper one to $\mathrm{U}=460-570 \mathrm{keV}$, with an energy resolution of $16 \%$.

We report results for a cylindrical phantoms of different size, initialised from a $\mu$-map with an error of $-20 \%$ in the inner cylinder w.r.t. the ground truth. Image size is $30 \times 30 \times 8$ with a voxel dimension of $1.2 \times 1.1 \times 3.25 \mathrm{~cm}^{3}$. Inner and outer ring diameters for the small cylinder are respectively 6 and $8 \mathrm{~cm}$ (Fig. 1). The big cylinder is 3 times bigger in the $x-y$ plane. In $\mathrm{z}$-dimension both cylinders are as long as the scanner $(26 \mathrm{~cm})$.

In the current results, attenuation masking was incorporated in the algorithm by only updating the $\mu$ values within the inner cylinder during MLTR-EB iterations.

\section{RESULTS AND DISCUSSION}

Results for the small cylinder show that the algorithm is able to compensate for errors due to the wrong assignment of population based density values. Fig. 1 reports the mean percentage error over iterations, for both the activity and the attenuation images, which converges to zero.

According to the findings in a previous study [2], we also decided to investigate the effects of the size of the phantom: 

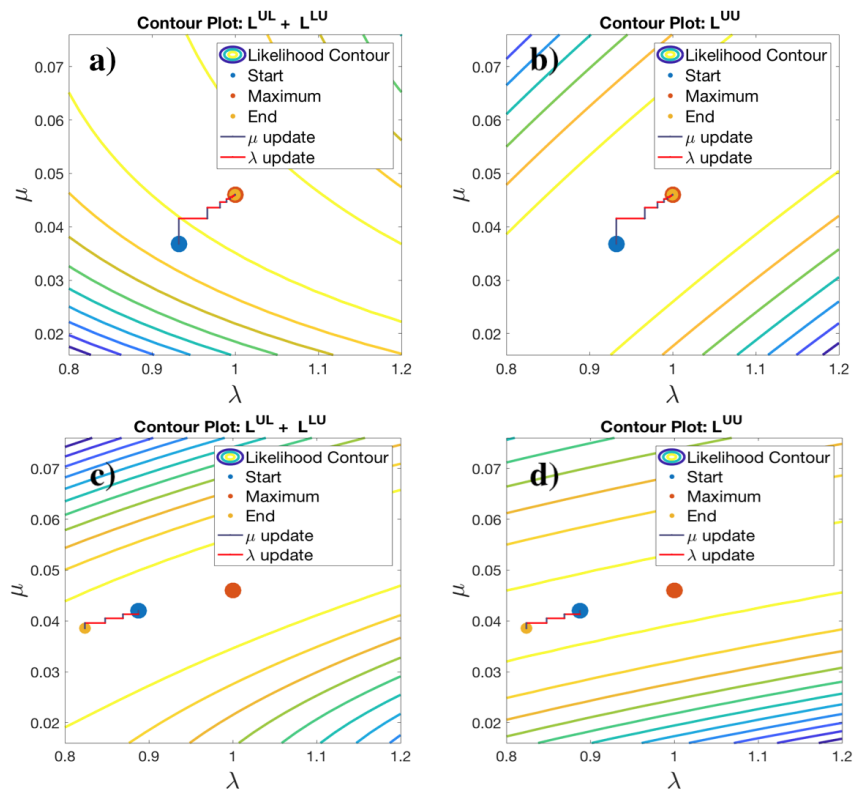

Fig. 2. First row: Contour plots related to the small cylinder, w.r.t the lower (a) and upper (b) energy window. Second row: Contour plots related to the big cylinder, w.r.t the lower (a) and upper (b) energy window.

a two-variable analysis was conducted on a larger cylinder of diameter $32 \mathrm{~cm}$. Only two constant values, activity and attenuation in the inner cylinder, were estimated at each iteration. This choice was made in order to reduce the complexity of the problem and isolate the number of variables that come into play.

Fig 2 shows the obtained activity and attenuation values for each iteration for the small (Fig. 2 a-b) and big (Fig. 2 c-d) cylinder, superimposed on the contour plots of the respective log-likelihoods.

This analysis shows that the size of the phantom affects the shape and inclination of the cost functions in the different energy windows. Furthermore, it shows that the algorithm behaviour depends on spatial scale: the algorithm converges to the true solution only for the small cylinder (Fig. 2 a-b).

\section{CONCLUSIONS}

Results from simulations demonstrated that the proposed MLAA-EB algorithm is capable of correctly estimating both the activity and the attenuation distribution for a small cylindrical phantom.

A subsequent study conducted to understand the effect of the size of the object demonstrated that the proposed approach is sufficient (and fast) for small phantoms, but insufficient for patient-scale objects.

We believe that the use of alternating optimisation between two different objective functions is the source of failure of this approach in the case of big objects. Therefore, future work will be oriented towards the development of a joint reconstruction algorithm that optimises both variables simultaneously.

Further efforts will have to be made to reconstruct the attenuation map without restricting the update to the lung region only. In addition, the proposed algorithm can be extended to include unscattered counts in the lower energy windows as well as multiple scatters. The impact of the presence of noise in the data will also have to be evaluated.

\section{ACKNOWLEDGEMENTS}

The authors would like to thank Dr. C.C. Watson (Siemens Healthineers) and Dr. Richard Brown (UCL) for helpful discussions.

\section{REFERENCES}

[1] Y. Berker and L. Yusheng, "Attenuation correction in emission tomography using the emission data - a review," Medical Physics, vol. 43, no. 2, pp. 807-832, 2016.

[2] Y. Berker, J. S. Karp, and V. Shulz, "Joint reconstruction of PET attenuation and activity from scattered and unscattered data," NSS/MIC Conference Record, 2017.

[3] L. Brusaferri, A. Bousse, N. Efthimiou, E. Emond, D. Atkinson, S. Ourselin, F. Brian, S. Arridge, and K. Thielemans, "Potential benefits of incorporating energy information when estimating attenuation from PET data," NSS/MIC Conference Record, 2017.

[4] C. C. Watson, D. Newport, and M. E. Casey, "A single scatter simulation technique for scatter correction in 3D PET."

[5] O. Klein and Y. Nishina, "'”er die Streuung von Strahlung durch freie Elektronen nach der neuen relativistischen Quantendynamik von Dirac"," Z. Phys., vol. 52, no. 11-12, pp. 853-869, 1929.

[6] R. H. Byrd, P. Lu, J. Nocedal, and C. Zhu, "A limited memory algorithm for bound constrained optimization," SIAM J. Sci. Comput., vol. 16, no. 5 , pp. 1190-1208, 1995.

[7] K. Thielemans, C. Tsoumpas, S. Mustafovic, T. Beisel, P. Aguiar, N. Dikaios, and M. W. Jacobson, "Stir: Software for tomographic image reconstruction release 2," Phys. Med. Biol, vol. 57, no. 4, pp. 867-883, 2012.

[8] A. M. Karlberg, O. Sæther, L. Eikenes, and P. E. Goa, "Quantitative comparison of PET performance-siemens biograph mCT and mMR," EJNMMI Phys., vol. 3, no. 5, p. 1, 2016. 